\title{
Three-Component Route for the Regioselective Construction of $\mathbf{N}$-Arylbenzimidazoles
}

benzimidazoles

o-chloroaryl

sulfonates

o-chloroaryl halides

\section{anilines}

amides

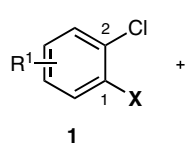

$$
+\mathrm{Ar}-\mathrm{NH}_{2}+\mathrm{R}^{2} \stackrel{\text { 少 }}{\mathrm{NH}_{2}} \stackrel{\begin{array}{c}
\text { catalyst }(2-6 \mathrm{~mol} \%) \\
\mathrm{Cs}_{2} \mathrm{CO}_{3}(2.4 \mathrm{equiv})
\end{array}}{t-\mathrm{BuOH}, 110^{\circ} \mathrm{C}, 12 \mathrm{~h}}
$$

$$
\text { (1 equiv) }
$$

(1.3 equiv)<smiles></smiles>

\section{8 examples}

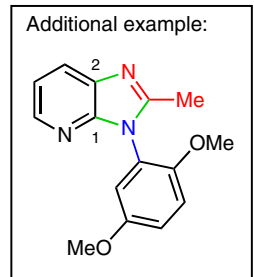

$\mathbf{X}=\mathrm{OTf}, \mathrm{Br}, \mathrm{C}$

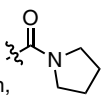

$\mathrm{R}^{1}=\mathrm{H}, 4-\mathrm{Me}, 5-\mathrm{Me}, 4-i-\mathrm{Pr}, 4-\mathrm{CF}_{3}$ 4-F, 4-OMe, 4-Ph, 5-CONHBn,

$\mathrm{R}^{2}=\mathrm{H}, \mathrm{Me}, \mathrm{Et}, c-\mathrm{Pr}, \mathrm{Cy}, \mathrm{CH}=\mathrm{CHMe}, \mathrm{Bn},(2-\mathrm{py}) \mathrm{CH}_{2}, \mathrm{Ph}, 3-\mathrm{F}_{3} \mathrm{CC}_{6} \mathrm{H}_{4}, 4-\mathrm{MeOC}_{6} \mathrm{H}_{4}$, thiophen-2-yl

$\mathrm{Ar}=3,5-\mathrm{Me}_{2} \mathrm{C}_{6} \mathrm{H}_{3}, 4-t-\mathrm{BuC}_{6} \mathrm{H}_{4}, 4-t-\mathrm{BuO}_{2} \mathrm{CC}_{6} \mathrm{H}_{4}, 4-\mathrm{F}_{3} \mathrm{CC}_{6} \mathrm{H}_{4}, 4-\mathrm{FC}_{6} \mathrm{H}_{4}, 2,4-\mathrm{F}_{2} \mathrm{C}_{6} \mathrm{H}_{3}, 2-\mathrm{MeOC}_{6} \mathrm{H}_{4}$

3-MeOC $\mathrm{H}_{4}, 4-\mathrm{MeOC}_{6} \mathrm{H}_{4}, 3-\mathrm{F}_{3} \mathrm{COC}_{6} \mathrm{H}_{4}, 4-\mathrm{F}_{3} \mathrm{COC}_{6} \mathrm{H}_{4}, 3,4-\left(\mathrm{OCH}_{2} \mathrm{O}\right) \mathrm{C}_{6} \mathrm{H}_{3}$, 3-py, 5-(2-MeOpy), z- $N$

$61 \%($ from $\mathrm{X}=\mathrm{Cl}$ )<smiles>COc1cccc(OC)c1</smiles>

$\mathrm{R}^{1}=i-\mathrm{Pr}, \mathrm{CF}_{3}, \mathrm{~F}, \mathrm{Ph}$ $\mathrm{R}^{2}=\mathrm{H}, \mathrm{Me}, \mathrm{C}-\mathrm{Pr}, \mathrm{Bn}$

$\mathrm{Ar}=2,4-\mathrm{F}_{2} \mathrm{C}_{6} \mathrm{H}_{3}, 3-\mathrm{F}_{3} \mathrm{COC}_{6} \mathrm{H}_{4}, 3,4-\left(\mathrm{OCH}_{2} \mathrm{O}\right) \mathrm{C}_{6} \mathrm{H}_{3}, 3-\mathrm{py}$

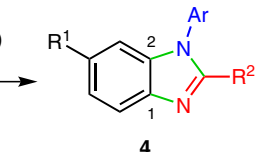

4

4 examples $64-81 \%$ yield

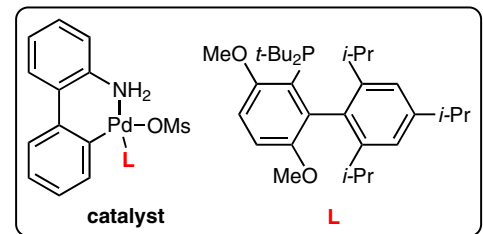

palladium catalysis

Significance: Reported is a regiocontrolled synthesis of $\mathbf{N}$-arylbenzimidazoles $\mathbf{3}$ and $\mathbf{4}$ via a threecomponent reaction using a palladium catalyst. In this reaction, the 2-chloroarenes $\mathbf{1}$ and $\mathbf{2}$ show different reactivities in combining with anilines and amides in sequential intermolecular amination and amidation reactions and cyclizations to regioselectively afford two types of $N$-arylbenzimidazoles, $\mathbf{3}$ and $\mathbf{4}$, in moderate to good yields. The reaction scope and limitations for the 2-chloroarenes 2 were not well investigated.
Comment: Benzimidazoles are often found in nature and in drugs displaying broad spectrum pharmaceutical activities (see Review below). Poor regiocontrolled benzimidazole arylations to give mixtures of isomeric products have been previously reported (e.g., D. Yang et al. J. Org. Chem. 2008, 73, 7841). The present method not only assembles the substituted benzimidazoles in a straightforward way from readily available starting materials, but also offers a high regiocontrol in the construction of single isomer products.

Review: F. Fei, Z. Zhuo Expert Opin. Ther. Patents 2013, 23, 1157-1179. 